\title{
Comparative Study Between Total Intravenous Anesthesia Versus Volatile Induction and Maintenance Anesthesia with Sevoflurane for Bariatric Operations Anwar Mohamed Mostafa Alhasanin, Mostafa Mohamed Mohamed El-Sayed, Mohamed Abd El Nasser Zaki Basiouny*
}

Anesthesiology and Intensive Care Department, Faculty of Medicine, Al-Azhar University

* Corresponding author: Mohamed Abd El Nasser Zaki Basiouny, E-mail: mohamedassassy@ yahoo.com

\begin{abstract}
Background: Morbid obesity is defined as a body weight $160 \%$ above the ideal weight for survival or $40 \mathrm{~kg} / \mathrm{m} 2$ or $>35$ with serious comorbidity. Long-term surveys revealed that surgical intervention has been the only method to effectively sustain weight loss in these patients.

Objectives: To compare total intravenous anesthesia applied with propofol-fentanyl to sevoflurane single breath and maintenance anesthesia.

Patients and Methods: This prospective randomized clinical study was approved by the ethics committee in AlAzhar University and a written informed patient's consent was obtained. This study included 50 patients of both sexes, admitted for bariatric surgery carried out at Al-Azhar University Hospitals (El-Hussein and Bab El-Sharia). They were randomly allocated into two equal groups, TIVA group: 25 patients will receive total intravenous anesthesia applied with propofol-fentanyl. Group VIMA: sevoflurane single breath induction and maintenance group: 25 patients will receive - sevoflurane single breath induction and maintenance.

Results: Total intravenous anesthesia using (fentanyl and propofol). Volatile induction and maintenance anesthesia (Inhalational anesthesia) using (fentanyl and sevoflurane). The following parameters were assessed between the 2 groups: Hemodynamics, SPo2 and ET Co2 were continuously monitored and recorded at 15 min interval.

Conclusion: This study showed that both TIVA and VIMA were effective in bariatric surgeries. Regarding neuroendocrine stress response, TIVA group was provided suppressed hormone levels suggesting that there was suppression of stress response unlike VIMA group that showed even raised serum levels of those hormones.

Keywords: total intravenous anesthesia, volatile induction, maintenance anesthesia, sevoflurane, bariatric operations.
\end{abstract}

\section{INTRODUCTION}

Obesity is defined as a body weight that is $160 \%$ higher than the ideal weight for survival or 40 $\mathrm{kg} / \mathrm{m} 2$ or $>35$ with serious joint morbidity. Longterm studies have revealed that surgical intervention is the only way to maintain the effectiveness of weight loss in these patients. Various processes have been developed to treat pathological obesity. The choice of anesthesia technique for general anesthesia in obese patients remains controversial ${ }^{(\mathbf{1})}$.

Many surgical procedures were designed to manage obese patient and were considered as a method of management of obesity. Obese patients have a challenge in dose calculations and adjustments due to different volume of distribution that indeed need accurate calculation for drugs to match weight ${ }^{(2)}$.

Sevoflurane has been suggested to be the long awaited, ideal inhalational anesthetic for its properties of being pleasant smelling, relatively nonirritating to the airways and its low blood-gas solubility which allows rapid induction and recovery from anesthesia ${ }^{(3)}$.

Clinicians have taken advantage of these attributes to adopt it for volatile induction and maintenance (VIMA), especially in the day surgery setting, since it has a potential to allow 'fast-tracking' of patients ${ }^{(4)}$. A contrasting but also popular technique for day surgery is total intravenous anesthesia (TIVA) with propofol which boasts rapid induction and recovery (5).

\section{AIM OF THIS STUDY}

To compare total intravenous anesthesia (TIVA) applied with propofol-fentanyl to sevoflurane single breath and maintenance anesthesia.

Also, to investigate hemodynamics, blood gases, stress response and recovery characteristics of the two different anesthetic techniques and determined convenient technique for bariatric surgery.

\section{PATIENTS AND METHODS}

- Ethical approval:

- This prospective randomized clinical study was approved by the ethics committee in Al-Azhar University and a written informed patient's consent was obtained.

- The study was carried out at Al-Azhar University Hospitals (El-Hussein and Bab El-Sharia).

- Patients:

- This study included 50 patients of both sexes, admitted for bariatric surgery carried out at AlAzhar University Hospitals (El-Hussein and Bab El-Sharia).

- They were randomly allocated into two equal groups, 25 patients each $(\mathrm{n}=25)$ :

- Group (TIVA): (TIVA) group: 25 Patients will receive total intravenous anesthesia applied with propofol-fentanyl.

- Group (VIMA) - sevoflurane single breath induction and maintenance group-: 25 patients 
will receive - sevoflurane single breath induction and maintenance.

Inclusion criteria: patients with:

1- American Society of Anesthesiologist (ASA) class I and II.

2- Aged 25-60 years of both sexes.

3- Undergoing bariatric surgery.

4- Time of operation ranges from 1-2 hours.

5- Body mass index from $35 \%-50 \%$

6- The patients had no splenic, lung, heart or liver disorders found on clinical and biochemical tests.

\section{Exclusion criteria:}

1. ASA $\geq$ class III.

2. Patients with a history of allergy to any of the medications used in the study.

3. Body mass index $(\mathrm{BMI})>35$.

4. Patients with bleeding disorders.

5. Patients on anticoagulant therapy or antiplatelets.

6. Patients who when forced to use pharmacological drug as nitroglycerin to control hypertension occurring during operation were excluded.

7. Time of operation not more than (2) hours.

8. Patient refusal.

\section{Preoperative evaluation:}

Patients of two groups were subjected to medical history and clinical examination of vital organs (heart, chest and revising labs for abnormality, also other exclusion criteria and also assessment of ASA physical status of the patients).The medications were determined after calculating corrected weight (corrected weight $=[0.4 \mathrm{x}$ excess weight $]+$ ideal weight). Before operation, $5 \mathrm{ml}$ sample of blood for measuring plasma cortisol level was taken

\section{The patients were screened for suitability by:}

- History of previous complications from general anesthesia.

- Physical examination: chest, heart and abdominal examination.

- Investigations: complete blood picture, coagulation profile, and chest X-ray.

\section{Methods:}

Group I (Receive total intravenous anesthesia):

Two I.V. cannula one in each arm was inserted, one for IV infusion pump and the other for IV fluid and induction of anesthesia.

All patients were pre-oxygenated via a face mask for 5 minutes prior to induction.

The infusion line was attached to the intravenous cannula from the start, after administering fentanyl $(1 \mathrm{mcg} / \mathrm{kg}$ i.v.) anesthesia was induced with propofol $2 \mathrm{mg} / \mathrm{kg}$. The infusion rate of propofol was 12 $\mathrm{mg} / \mathrm{kg} / \mathrm{hr}$ for $10 \mathrm{~min}$ following intubation, then 10 $\mathrm{mg} / \mathrm{kg} / \mathrm{hr}$ for next $10 \mathrm{~min}$ and continued at 6-10 $\mathrm{mg} / \mathrm{kg} / \mathrm{hr}$, the infusion rate was adjusted according to hemodynamic responses to maintain a mean arterial pressure (MAP) not below 35\% of the base and fentanyl infusion at a rate of $0.1-0.5 \mu \mathrm{g} / \mathrm{kg} / \mathrm{h}$. After loss of the eyelash reflex, administration of cisatracurium $0.15 \mathrm{mg} / \mathrm{kg}$ IV was done and the intubation was done, oxygen flow rate was adjusted to $3 \mathrm{~L} / \mathrm{min}$ and controlled mechanical ventilation started.

Anesthesia was maintained by continuous infusion of propofol and fentanyl using a specific syringe infusion pumps (Injectomat Agilia, Fresenius Kabi, Syringe Pump, made in France) previously prepared with two $50 \mathrm{cc}$ syringes containing $500 \mathrm{mg}$ thick propofol and $50 \mu \mathrm{g}$ of fentanyl in $50 \mathrm{cc}$ normal saline.

Group II (Receive an inhalational anesthesia):

All patients were pre-oxygenated via a face mask for 5 minutes prior to induction. The face mask was placed over the nose and mouth and while breathing $100 \%$ oxygen from the breathing system, the patients were asked to take a deep breath and exhale to tidal volume with sevoflurane vaporizer on MAC 2 vol\%. The patients were asked to open their eyes every 3-5 seconds. At loss of the eyelash reflex, a bolus dose of fentanyl $1 \mu \mathrm{g} / \mathrm{kg}$, followed by cisatracurium 0.15 $\mathrm{mg} / \mathrm{kg}$ and the intubation was done, oxygen flow rate was adjusted to $3 \mathrm{~L} / \mathrm{min}$ and controlled mechanical ventilation started.

Anesthesia was maintained by Sevoflurane inhalation (2-4 vol\%), and continuous infusion of fentanyl using a specific syringe infusion pump (Injectomat Agilia, Fresenius Kabi, Syringe Pump, made in France) previously prepared with $50 \mathrm{cc}$ syringe containing $50 \mu \mathrm{g}$ of fentanyl in $50 \mathrm{cc}$ normal saline. The infusion rate of fentanyl was 0.1-0.5 $\mu \mathrm{g} / \mathrm{kg} / \mathrm{h}$, from the start in a separate IV line and the concentration of sevoflurane was adjusted according to hemodynamic responses to maintain MAP not below $35 \%$ of the base.

\section{In both groups:}

Mechanical ventilation was adjusted to maintain end-tidal $\mathrm{CO}_{2}$ between 35 and $40 \mathrm{mmHg}$. During intraoperative period, both groups received 6-10 $\mathrm{ml} / \mathrm{kg} / \mathrm{h}$ Crystalloids as a maintenance. Muscle relaxation was maintained with intermittent boluses of cisatracurium guided by nerve stimulator. The volume of intraoperative blood loss was estimated based on the volume of blood in the suction bottle and the number of soaked gauze pads and was replaced.

At the end of surgery, all anesthetic agents were stopped, and neuromuscular blocking was reversed by neostigmine $0.04-0.08 \mathrm{mg} / \mathrm{kg}$ IV and atropine $0.02-0.04 \mathrm{mg} / \mathrm{kg} \mathrm{IV}$.

Patients were asked repeatedly in a normal tone of voice to open their eyes until an appropriate response was obtained. The trachea was extubated when a regular spontaneous breathing pattern had been re-established. The following recovery times 
were recorded, time to eye opening, verbal communication and time to orientation.

After surgery, all patients were transferred to a postanesthesia care unit (PACU).

\section{Statistical analysis}

Recorded data were analyzed using the statistical package for social sciences, version 20.0 (SPSS Inc.,
Chicago, Illinois, USA). Quantitative data were expressed as mean \pm standard deviation (SD). Qualitative data were expressed as frequency and percentage. Probability (P-value): $\mathrm{P}$-value $<0.05$ was considered significant, $\mathrm{P}$-value $<0.001$ was considered as highly significant, P-value $>0.05$ was considered insignificant.

\section{RESULTS}

Table (1): Comparison between the two groups according to demographic data.

\begin{tabular}{|c|c|c|c|c|}
\hline Demographic Data & Group TIVA (N=25) & Group VIMA $(\mathrm{N}=25)$ & $\mathrm{t} / \mathbf{x} 2 \#$ & p-value \\
\hline \multicolumn{5}{|l|}{ Age (years) } \\
\hline Mean \pm SD & $27.45 \pm 7.25$ & $27.92 \pm 7.49$ & 0.037 & 0.722 \\
\hline \multicolumn{5}{|l|}{ Sex } \\
\hline Male & $9(36.0 \%)$ & $10(40.0 \%)$ & \multirow{2}{*}{$0.123 \#$} & \multirow{2}{*}{0.625} \\
\hline Female & $16(64.0 \%)$ & $15(60.0 \%)$ & & \\
\hline \multicolumn{5}{|l|}{ BMI [wt/(ht)^2] } \\
\hline Mean \pm SD & $43.78 \pm 6.57$ & $44.29 \pm 6.64$ & 0.016 & 0.760 \\
\hline \multicolumn{5}{|l|}{ ASA } \\
\hline $\mathrm{I}$ & $21(84 \%)$ & $20(80 \%)$ & \multirow{2}{*}{$0.263 \#$} & \multirow{2}{*}{0.532} \\
\hline II & $4(16 \%)$ & $5(20 \%)$ & & \\
\hline
\end{tabular}

t-Independent Sample t-test; $\mathrm{x}^{2}$ : Chi-square test

p-value $>0.05 \mathrm{NS}$; *p-value <0.05 S; **p-value <0.001 HS

Demographic data of the two groups of patients showed no statistically significant differences as regard age, sex, weight, height and ASA state $(\mathrm{P}>0.05)$ as shown in the Table (1).

Table (2): Comparison between the two groups according to the time of recovery (min).

\begin{tabular}{|c|c|c|c|c|}
\hline Recovery profile & Group TIVA $(\mathrm{N}=25)$ & Group VIMA $(\mathrm{N}=25)$ & t-test & p-value \\
\hline Open eyes(Minutes) & & & \multirow{2}{*}{8.854} & \multirow{2}{*}{$<0.001 * *$} \\
\hline Mean \pm SD & $9.49 \pm 1.21$ & $8.46 \pm 0.94$ & & \\
\hline Verbal communication(Minutes) & & & \multirow{2}{*}{28.929} & \multirow{2}{*}{$<0.001 * *$} \\
\hline Mean \pm SD & $12.52 \pm 1.00$ & $10.69 \pm 1.13$ & & \\
\hline Mental orientation(Minutes) & & & \multirow{2}{*}{35.484} & \multirow{2}{*}{$<0.001 * *$} \\
\hline Mean \pm SD & $15.20 \pm 0.95$ & $13.28 \pm 1.05$ & & \\
\hline
\end{tabular}

t-Independent Sample t-test

$*$ p-value $<0.05 \mathrm{~S}$; **p-value $<0.001 \mathrm{HS}$

There was statistically significant difference between the two groups according to recovery profile as regard time to eye opening, verbal communication and mental orientation as shown in Table (2).

Table (3): Comparison between the two groups according to postoperative nausea and vomiting.

\begin{tabular}{|c|c|c|c|c|}
\hline Post-Operative Nausea and Vomiting & Group TIVA (N=25) & Group VIMA (N=25) & x2 & p-value \\
\hline No & $22(88 \%)$ & $17(68 \%)$ & \multirow{2}{*}{4.033} & $0.041 *$ \\
\hline Yes & $3(12 \%)$ & $8(32 \%)$ & 4 \\
\hline
\end{tabular}

$\mathrm{x} 2$ : Chi-square test; *p-value $<0.05 \mathrm{~S}$

There was statistically significant difference between the two groups according to post operative nausea and vomiting as shown in Table (3).

Table (4): Comparison between the two groups according to norepinephrine (pg/ml).

\begin{tabular}{|c|c|r|c|c|}
\hline Norepinephrine (pg/ml) & Group TIVA (N=25) & Group VIMA (N=25) & t-test & p-value \\
\hline Day before surgery (T0) & $252.5 \pm 42.9$ & $262.6 \pm 44.6$ & 1.214 & 0.224 \\
Before induction of anesthesia (T1) & $121.2 \pm 20.6$ & $404.0 \pm 68.7$ & 4.069 & $0.009 *$ \\
After intubation (T2) & $308.1 \pm 52.4$ & $383.8 \pm 65.2$ & 1.337 & 0.337 \\
First day post operative (T3) & $131.3 \pm 22.3$ & $204.5 \pm 34.8$ & 4.324 & $0.007 *$ \\
\hline
\end{tabular}

t-Independent Sample t-test; p-value $>0.05 \mathrm{NS}$

There was statistically significant difference between the two groups according to norepinephrine (pg/ml) at T1 and T3 as shown in Table (4). 
Table (5): Comparison between the two groups according to epinephrine (pg/ml).

\begin{tabular}{|c|c|c|c|c|}
\hline Epinephrine (pg/ml) & $\begin{array}{c}\text { Group } \\
\text { TIVA } \\
(\mathbf{N}=\mathbf{2 5})\end{array}$ & $\begin{array}{c}\text { Group VIMA } \\
(\mathbf{N}=\mathbf{2 5})\end{array}$ & t-test & p-value \\
\hline Day before surgery (T0) & $47.5 \pm 8.1$ & $48.5 \pm 8.2$ & 0.501 & 0.610 \\
Before induction of anesthesia (T1) & $25.3 \pm 4.3$ & $181.8 \pm 30.9$ & 4.199 & $<0.001^{* *}$ \\
After intubation (T2) & $28.3 \pm 4.8$ & $90.9 \pm 15.5$ & 5.537 & $<0.001^{* *}$ \\
First day post operative (T3) & $29.8 \pm 5.1$ & $48.5 \pm 8.2$ & 4.155 & $0.008^{*}$ \\
\hline
\end{tabular}

t-Independent Sample t-test; p-value >0.05 NS

There was statistically significant difference between the two groups according to epinephrine (pg/ml) at $\mathrm{T} 1, \mathrm{~T} 2$ and $\mathrm{T} 3$ as shown in Table (5).

Table (6): Comparison between the two groups according to cortisol $(\mu \mathrm{g} / \mathrm{dl})$.

\begin{tabular}{|c|c|c|c|c|}
\hline Cortisol (microgr/dI) & Group TIVA (N=25) & Group VIMA (N=25) & t-test & p-value \\
\hline Day before surgery (T0) & $9.1 \pm 1.5$ & $9.1 \pm 1.5$ & 1.492 & 0.317 \\
Before induction of anesthesia (T1) & $7.1 \pm 1.2$ & $24.2 \pm 4.1$ & 4.619 & $<0.001^{* *}$ \\
After intubation (T2) & $6.1 \pm 1.0$ & $28.3 \pm 4.8$ & 6.091 & $<0.001^{* *}$ \\
First day post operative (T3) & $7.3 \pm 1.2$ & $13.1 \pm 2.2$ & 4.570 & $0.011^{*}$ \\
\hline
\end{tabular}

t-Independent Sample t-test; $p$-value $>0.05$ NS

This table shows statistically significant difference between the two groups according to cortisol at T1, T2 and T3 as shown in Table (6).

\section{DISCUSSION}

Obesity is a major health problem affecting every organ system and is associated with many health consequences. Many surgical procedures were designed to manage obese patient and is considered as a method of management of obesity. Obese patients have a challenge in dose calculations and adjustments due to different volume of distribution that indeed need accurate calculation for drugs to match weight ${ }^{(2)}$.

Sevoflurane-based inhalational anesthetic technique can be practiced wherever a general anesthetic is given. However, there is always a need to explore newer techniques and drugs to try and achieve better results and conditions for surgeries like bariatric surgeries. One such technique that is gaining tremendous popularity for controlled hypotension is TIVA with propofol and opioids ${ }^{(6)}$.

This study was designed to evaluate TIVA with propofol and fentanyl and to determine whether better results and operative conditions can be achieved when compared to conventional sevoflurane-based inhalational anesthetic technique for bariatric surgeries.

Sevoflurane is widely used in clinical anesthesia because of its relative lack of airway irritation and myocardial depressant effect. Sevoflurane has a low blood gas partition coefficient of 0.65 , which contributes to more rapid induction of and emergence from anesthesia than with other volatile anesthetics in current clinical practice ${ }^{(7)}$.

In the present study, addition of fentanyl infusion to propofol infusion (group I) or sevoflurane inhalation (group II) was used for maintenance of anesthesia.
In the present work, there were no statistically significant difference between the two groups in demographic data, including: age, sex, BMI, ASA group for our study for bariatric surgeries.

According to hemodynamic parameters, it was found that, there were no statistically significant difference between the two groups in hemodynamics (systolic, diastolic, mean arterial pressure and heart rate), with slight decrease in TIVA group than VIMA group. The lower HR observed during TIVA requires careful interpretation because fentanyl had been infused continuously along with propofol. The lower heart rate might also have been because of opioids such as fentanyl. However, the infused fentanyl dose in both groups was the same, which suggests that the lower HR in TIVA group was related to propofol. Although many obese patients have history of HTN, its effect was not significant on the data collected as we choose controlled patients.

Both propofol and inhalation agents have a vasodilatory effect in a concentration-dependent manner. However, the extent of reflex tachycardia is quite variable. Compared with the apparent reflex tachycardia in isoflurane, sevoflurane usually does not alter the heart rate ${ }^{(7)}$. In contrast, propofol inhibits the baroreflex and can even result in bradycardia ${ }^{(8)}$. Therefore, propofol suppresses the cardiac output more than sevoflurane ${ }^{(9)}$. So, in the patients that did not have a cardiovascular disease and the MBP was controlled within the same range such as in the present study, the heart rate was lower in the TIVA than in the inhalational anesthesia.

Ledowski et al. ${ }^{(10)}$, reported that sevofluranebased anesthesia resulted in higher HRs and higher serum levels of epinephrine, norepinephrine, 
adrenocorticotropic hormone (ACTH) and cortisol than propofol-based TIVA and suggested that TIVA provided stronger inhibition of the neuroendocrine stress than sevoflurane. The other reason for the higher HR related to sevoflurane could be because of its vagolytic effect ${ }^{(\mathbf{1 1})}$.

In terms of MAP, there was no significant difference between the two groups at the time points of intubation, the beginning of operation, during maintenance of anesthesia, but TIVA provided a lower MAP than sevoflurane anesthesia. This could be explained by the different effect of the two techniques on neuroendocrine stress.

TIVA and sevoflurane provided a similar depth of anesthesia to maintain MAP at the same level, but because of stronger inhibition of the neuroendocrine reaction to stress by TIVA the MAP was lower with TIVA than with VIMA during operation ${ }^{(\mathbf{1 0})}$.

According to ETCo2 and $\mathrm{SpO}_{2}$, in our study there were no significant changes in their levels throughout the surgery in both groups.

In the present study, the recovery profile showed a statistically significant difference between the two groups, with faster recovery in the Sevoflurane group.

Our results were similar to a study done by Orhon et al. ${ }^{(12)}$ and Kumar et al. ${ }^{(13)}$, who also found that recovery time after sevoflurane anesthesia was shorter than with propofol based anesthesia.

Shah and Adaroja ${ }^{(6)}$, compared the emergence and postoperative recovery profile between sevoflurane and propofol. They found that sevoflurane has a better recovery profile than the intravenous propofol. This effect of sevoflurane has been attributed to its insoluble nature, rapid wash in and out and low blood: gas partition coefficient, all of which lead to the rapid emergence.

The results of this study agreed with Castagnini et al. (14), who studied the differences between sevoflurane and propofol regarding recovery criteria in the neuroradiological procedures and found that use of sevoflurane for maintenance of anesthesia for prolonged neurosurgical procedures is associated with more rapid recovery than propofol.

On the other hand, these results were inconsistent with those of Ebert et al. (15), who studied the difference in recovery between the sevoflurane anesthesia to that of isoflurane and propofol anesthesia in surgeries of intermediate duration of all age groups and found that the recovery from sevoflurane was three to four minutes faster than that with isoflurane in all age groups but there was no significant difference in recovery times between sevoflurane and propofol.

At the same time this study was also inconsistent with study done by Sneyd et al. ${ }^{(16)}$, they found that both sevoflurane and propofol in combination with remifentanil, are satisfactory agents for maintenance of anesthesia in neurosurgical patients and the differences in the recovery times were not clinically significant.

According to postoperative nausea and vomiting. In our study there were a statistically significant difference between the two groups.

In a review of early postoperative complications following neurosurgical procedures, PONV was the commonest complication (38.7\%), although the anesthesia technique was not mentioned in that study (17).

In the present study six patients $(30 \%)$ from VIMA group developed nausea and vomiting in the post-operative period, while only two patients in TIVA group (10\%) had nausea and vomiting.

Propofol appears to possess antiemetic properties that contribute to a lower incidence of emetic sequelae after its use. In fact, subanesthetic doses of propofol (10-20 mg) have also been successfully used to treat nausea and emesis in the early postoperative period ${ }^{(\mathbf{1 8})}$.

The postulated mechanisms of propofol antiemetic effect may include; antidopaminergic activity, depressant effect on the chemoreceptor trigger zone and vagal nuclei, decreased release of glutamate and aspartate in the olfactory cortex, and reduction of serotonin concentrations in the area postrema ${ }^{(\mathbf{1 9})}$.

The results of this study were in line with the results of Gupta et al. ${ }^{(20)}$, who reported that TIVA with propofol has been found by to produce less PONV compared to inhalational anesthesia in nonneurosurgical patients.

As regard neuroendocrine stress response, our study showed that there was significant statistical difference between TIVA and sevoflurane-based anesthesia.

Ledowski et al. ${ }^{(10)}$, reported that sevofluranebased anesthesia resulted in higher HRs and higher serum levels of epinephrine, norepinephrine, adrenocorticotropic hormone (ACTH) and cortisol than propofol-based TIVA and suggested that TIVA provided stronger inhibition of the neuroendocrine stress than sevoflurane. The other reason for the higher HR related to sevoflurane could be because of its vagolytic effect ${ }^{(\mathbf{1 1})}$.

In general, VIMA has the best recovery profile and less hemodynamic effect but stress response was better and significantly suppressed with TIVA.

\section{CONCLUSION}

This study showed that both TIVA and VIMA were effective in bariatric surgeries. Regarding neuroendocrine stress response, TIVA group was provided suppressed hormone levels suggesting that there was suppression of stress response unlike VIMA group that showed even raised serum levels of those hormones. 
Regarding the recovery criteria, VIMA group provided faster recovery than TIVA group. Regarding postoperative nausea and vomiting, TIVA group significantly reduces the incidence of postoperative nausea and vomiting, in the early recovery period than VIMA group.

\section{REFERENCES}

1. Renquist K (2002): Obesity classification. Obes. Surg., 7(6):523.

2. Apovian CM, Aronne LJ, Bessesen DH et al. (2015): Pharmacological management of obesity: an endocrine Society clinical practice guideline. J Clin Endocrinol. Metab., 100(2):342-62.

3. Balsiger BM, Murr MM, Poggio JL et al. (2008): Bariatric surgery: surgery for weight control in patients with morbid obesity. Med Clin North Am., 84:477-89.

4. Derzie AJ, Silvestri F, Liriano E et al. (2000): Wound closure technique and acute wound complications in gastric surgery for morbid obesity: a prospective randomized trial. J Am Coll Surg., 191:238-43.

5. Kakkar VV, Howe CT, Nicolaides AN et al. (2009): Deep vein thrombosis of the leg: is there a "high risk" group? Ann Surg., 120:527-32.

6. Shah A and Adaroja RN (2011): Comparison of hemodynamic changes with propofol and sevoflurane anesthesia during laparoscopic surgery. National journal of medical research, 1(2): $76-79$.

7. Larson CP and Jaffe RA (2016): The Best Volatile Anesthetic Ever Developed. Springer International Publishing. Practical Anesthetic Management, 91-102.

8. Reves J, Glass $P$ and McEvoy $M$ (2005): Intravenous nonopioid anesthetics. In: Miller RD, ed., Miller's Anesthesia, Philadelphia; Elsevier Churchill Livingstone, 323-4.

9. Husedzinovic I, Tonkovic D, Barisin $\mathbf{S}$ and Bradic $\mathbf{N}$ et al. (2003): Hemodynamic differences in sevoflurane versus propofol anesthesia. Coll Antropol., 27: 205-12.

10. Ledowski $T$, Bein $B$ and Hanss $R$ (2005): Neuroendocrine stress response and heart rate variability: a comparison of total intravenous versus balanced anesthesia. Anesth Analg., 101:1700-1705.
11. Petersen KD, Landsfeldt U, Cold GE and Petersen CB et al. (2003): Intracranial pressure and cerebral hemodynamic in patients with cerebral tumors. Anesthesiology, 98:329-36.

12. Orhon ZN, Devrim $S$ and Celik $M$ (2013): Comparison of recovery profiles of propofol and sevoflurane anesthesia with bispectral index monitoring in percutaneous nephrolithotomy. Korean J Anesthesiol., 64 (3): 223 - 228.

13. Kumar A, Vasanthan MR and Kannan N (2013): Comparison of recovery from propofol TIVA and sevoflurane VIMA in day case surgeries. Journal of pharmaceutical and biomedical sciences, 31(31): 121420.

14. Castagnini HE, Van Eijs F, Salevsky FC and Nathanson MH (2004): Sevoflurane for interventional neuroradiology procedure is associated with more rapid early recovery than propofol. Can J Anesth., 51:5:486491.

15. Ebert TJ, Robinson BJ, Uhrich TD and Mackenthum A et al. (1998): Recovery from sevoflurane anesthesia: a comparison to isoflurane and propofol anesthesia. Anesthesiology, 89: 1524-1531.

16. Sneyd JR, Andrew CJ and Tsubokawa T (2005): Comparison of propofol/remifentanyl and sevoflurane/remifentanyl for maintenance of anesthesia for elective intracranial surgery. Br J Anesth., 94: 778783.

17. Gan T, Meyet T, Apfel C and Chung F (2003): Consensus guidelines for managing postoperative nausea and vomiting. Anesth Analg., 97(1): 62-71.

18. Gan T, Glass $P$ and Howell S (1997): Determination of plasma concentrations associated with $50 \%$ reduction in postoperative nausea. Anesthesiology, 87:779.

19. Jen WC and Paul FW (2001): Propofol antiemetic properties. Nonopioid Intravenous Anesthesia. Clinical Anesthesia; 4th edition. Churchil Livingstone. New York.

20. Gupta A, Stierer T and Zuckerman R (2004): Comparison of recovery profile after ambulatory anesthesia with propofol, isoflurane, sevoflurane and desflurane: a systematic review. Anesth Analg., 98(3): 632-641. 